\title{
Adjuvant TKI treatment of EGFR-mutant lung cancer-already ripe for decision?
}

\author{
Diego Kauffmann-Guerrero
}

Division of Respiratory Medicine and Thoracic Oncology, Department of Internal Medicine V and Thoracic Oncology Centre Munich, University of Munich (LMU), Comprehensive Pneumology Center, Member of the German Center for Lung Research (DZL), Munich, Germany

Correspondence to: Dr. Diego Kauffmann-Guerrero. Hospital of University of Munich (LMU), Ziemssenstraße 1, 80336 Munich, Germany.

Email: Diego.KauffmannGuerrero@med.uni-muenchend.de.

Provenance and Peer Review: This article was commissioned by the editorial office, Translational Lung Cancer Research. The article did not undergo external peer review.

Comment on: Liang W, Cai K, Chen C, et al. Society for Translational Medicine consensus on postoperative management of EGFR-mutant lung cancer (2019 edition). Transl Lung Cancer Res 2019;8:1163-73.

Submitted Mar 24, 2020. Accepted for publication Apr 07, 2020.

doi: $10.21037 /$ tlcr.2020.04.13

View this article at: http://dx.doi.org/10.21037/tlcr.2020.04.13

Worldwide, lung cancer is the leading cause of cancerrelated deaths (1). Still, most patients are diagnosed with advanced disease and only few patients are eligible for curative treatment. Nevertheless, prognosis after "curative" surgery remains limited due to common relapse. After 5 years a third of stage I and up to $50 \%$ of stage II patients relapse (2). Better staging and new surgical techniques led to only a slight improvement of these historic data. Most of operated NSCLC patients relapse with distant metastases rather than local (3). Thus, common theory is, that undetectable micro-metastases were already present at the time of surgery and lead to tumor relapse during the course of follow-up.

Adjuvant chemotherapy is thought to fight these micrometastases to improve patients' outcome. Four cycles of adjuvant platinum-based chemotherapy had shown to expand survival of lung cancer patients especially with lymph node involvement and large tumors. However, gain of overall survival after 5 years is only $5.4 \%$ (4). As EGFR TKIs are highly effective in stage IV EGFR mutant NSCLC patients (5-8), it was quite reasonable to evaluate if EGFR positive patients will also benefit from adjuvant TKI treatment.

All together there are several studies investigating the role adjuvant EGFR TKI treatment: the BR19 study randomized completely resected stage IB-III NSCLC patients to receive gefitinib or placebo for 2 years. The patients were not selected regarding their EGFR mutation status. No benefit of disease-free survival (DFS) and overall survival (OS) was found neither in the total study cohort nor in the 15 patients with EGFR mutation (9).

In the RADIANT trial, Kelly et al. randomized 973 patients with surgically resected stage IB-IIIA NSCLC with EGFR expression or amplification and stratified patients with EGFR mutations. Patients received adjuvant treatment with erlotinib or placebo. The investigators found no benefit in DFS in patients with EGFR expression or EGFR mutation (10).

The EVAN study compared resected stage IIIA EGFR positive patients treated either with erlotinib for 1 year or vinorelbine plus cisplatin. DFS at 2 years was significantly expanded in the erlotinib group (81.4\% vs. 44.6\%) (11).

In the ADJUVANT trial, patients with stage II-IIIA EGFR-mutant NSCLC were treated after surgery with gefitinib over 2 years and compared with a cohort treated with 4 cycles of classic adjuvant chemotherapy. The median DFS was significantly longer in the gefitinib group (28.7 vs. 18 months) and the risk of relapse decrease by $40 \%$ (12).

Beside these randomized trials, there are some other non-randomized studies focusing on this topic. D'Angelo et al. examined a cohort of resected stage I-III EGFRmutant patients treated either with gefitinib or erlotinib after surgery. They found clues for improvement of DFS (13). Similar results have been reported by Janjigian and 
colleagues. The found a 2-year DFS benefit ( $89 \%$ vs. $72 \%$ ) in stage I to III patients treated with gefitinib or erlotinib after complete resection (14). The SELCET trial matched 100 patients with EGFR mutant NSCLC and resected stage IA to IIIA with a historic cohort and treated them with erlotinib for 2 years. DFS after 2 and 5 years was $88 \%$ and $56 \%$ respectively which was better than in the historic non treated cohort (15).

Recently, a consensus paper on postoperative management of EGFR-mutant lung cancer was published in Translational Lung Cancer Research by an Chinese committee of surgeons and oncologists (16). The authors proposed 8 consensuses as follows:

(I) EGFR testing is routinely recommended in all resected non-squamous NSCLC.

(II) Comprehensive predictive and prognostic markers an scores should be used to predict the risk of recurrence.

(III) Adjuvant EGFR-TKI treatment can prolong DFS compared with standard chemotherapy and can serve as one adjuvant therapy for patients with stage II-IIIA EGFR-mutant NSCLC.

(IV) Adjuvant chemotherapy, EGFR-TKI treatment and combination of both approaches can offered to EGFR positive NSCLC patients.

(V) The adjuvant TKI therapy should last at least 2 years.

(VI) Patients with activating EGFR mutation show higher risk of recurrence with brain and bone metastases compared to wild-type patients. Brain MRI and bone scans are recommended annually in addition to regular chest CT.

(VII) The result of genetic testing from initial tumor material could serve as reference in relapsed patients; alternatively, re-biopsy may confirm the gene mutation status and guide further therapy. Liquid biopsy, if available, is also a possible method for detection of EGFR mutation.

(VIII) EGFR-positive NSCLC patients relapsing after surgery, can be treated with EGFR-TKI (preferably osimertinib). In patients, who received adjuvant TKI treatment, re-challenge with EGFR -TKIs is possible. Presence of T790M mutations should be evaluated in re-biopsy specimens.

Despite the promising results of the ADJUVANT study, the data situation is currently not conclusive enough to recommend an adjuvant TKI treatment for EGFRmutant NSCLC patients. Given the above-mentioned recommendations there are several important points to consider: first of all, the ADJUVANT trial and the EVAN study could demonstrate a DFS advantage for TKI treated patients, but data on overall survival are still missing and the results were not confirmed by the RADIANT trial. Furthermore, the study population comprises only Asian patients of whom we know that they harbour a different EGFR biology than Caucasian patients. Thus, randomized studies also including Caucasian patients and data on overall survival are needed to give a general recommendation for adjuvant TKI treatment. Moreover, EGFR positive NSCLC patients are usually very sensitive to chemotherapy and irradiation, so that studies combining chemotherapy, radiotherapy and TKIs are necessary to evaluate the optimal adjuvant treatment for this cohort.

Regarding the duration of adjuvant TKI therapy: as we know from stage IV patients, TKI treatment dose not cure EGFR positive lung cancer, stopping the adjuvant treatment after 2 years might lead to just postpone the problem. Therefore, it will be crucial to see OS data. Nevertheless, EGFR testing should be performed in all resected nonsquamous NSCLC, not to guide an adjuvant treatment at present, but not to lose time in the case of relapse and to establish a risk adapted follow-up with special attention to brain and bone metastases.

There are no data for treatment recommendations in patients how relapse after adjuvant TKI treatment. Renewed EGFR testing would be indispensable in this condition to guide treatment decision. There are no data preferring a distinct TKI in this situation.

In summary, there are promising data that EGFRmutant NSCLC patients might benefit from adjuvant TKI treatment. Nevertheless, at present data are too weak to give a recommendation for this treatment option, especially in Caucasian patients. However, many open questions raised from the current studies. Accordingly, we need more conclusive data, especially on OS to elucidate the role of adjuvant TKI treatment in EGFR positive NSCLC.

\section{Acknowledgments}

Funding: None.

\section{Footnote}

Conflicts of Interest: The author has completed the ICMJE uniform disclosure form (available at http://dx.doi. org/10.21037/tlcr.2020.04.13). DKG reports personal fees 
from Roche, from Pfizer, from Boehringer Ingelheim, from AstraZeneca, outside the submitted work.

Ethical Statement: The author is accountable for all aspects of the work in ensuring that questions related to the accuracy or integrity of any part of the work are appropriately investigated and resolved.

Open Access Statement: This is an Open Access article distributed in accordance with the Creative Commons Attribution-NonCommercial-NoDerivs 4.0 International License (CC BY-NC-ND 4.0), which permits the noncommercial replication and distribution of the article with the strict proviso that no changes or edits are made and the original work is properly cited (including links to both the formal publication through the relevant DOI and the license). See: https://creativecommons.org/licenses/by-nc-nd/4.0/.

\section{References}

1. Riaz SP, Luechtenborg M, Coupland VH, et al. Trends in incidence of small cell lung cancer and all lung cancer. Lung Cancer 2012;75:280-4.

2. Nesbitt JC, Putnam JB, Jr., Walsh GL, et al. Survival in early-stage non-small cell lung cancer. Ann Thorac Surg 1995;60:466-72.

3. Boyd JA, Hubbs JL, Kim DW, et al. Timing of local and distant failure in resected lung cancer: implications for reported rates of local failure. J Thorac Oncol 2010;5:211-4.

4. Pignon JP, Tribodet H, Scagliotti GV, et al. Lung adjuvant cisplatin evaluation: a pooled analysis by the LACE Collaborative Group. J Clin Oncol 2008;26:3552-9.

5. Maemondo M, Inoue A, Kobayashi K, et al. Gefitinib or Chemotherapy for Non-Small-Cell Lung Cancer with Mutated EGFR. N Engl J Med 2010;362:2380-8.

6. Rosell R, Carcereny E, Gervais R, et al. Erlotinib versus standard chemotherapy as first-line treatment for European patients with advanced EGFR mutation-positive non-small-cell lung cancer (EURTAC): a multicentre, open-label, randomised phase 3 trial. Lancet Oncol 2012;13:239-46.

Cite this article as: Kauffmann-Guerrero D. Adjuvant TKI treatment of EGFR-mutant lung cancer-already ripe for decision? Transl Lung Cancer Res 2020;9(4):964-966. doi: 10.21037/tlcr.2020.04.13
7. Sequist LV, Yang JCH, Yamamoto N, et al. Phase III Study of Afatinib or Cisplatin Plus Pemetrexed in Patients With Metastatic Lung Adenocarcinoma With EGFR Mutations. J Clin Oncol 2013;31:3327.

8. Soria JC, Ohe Y, Vansteenkiste J, et al. Osimertinib in Untreated EGFR-Mutated Advanced Non-Small-Cell Lung Cancer. N Engl J Med 2018;378:113-25.

9. Goss GD, O'Callaghan C, Lorimer I, et al. Gefitinib versus placebo in completely resected non-small-cell lung cancer: results of the NCIC CTG BR19 study. J Clin Oncol 2013;31:3320-6.

10. Kelly K, Altorki NK, Eberhardt WE, et al. Adjuvant Erlotinib Versus Placebo in Patients With Stage IBIIIA Non-Small-Cell Lung Cancer (RADIANT): A Randomized, Double-Blind, Phase III Trial. J Clin Oncol 2015;33:4007-14.

11. Yue D, Xu S, Wang Q, et al. Erlotinib versus vinorelbine plus cisplatin as adjuvant therapy in Chinese patients with stage IIIA EGFR mutation-positive non-small-cell lung cancer (EVAN): a randomised, open-label, phase 2 trial. Lancet Respir Med 2018;6:863-73.

12. Zhong WZ, Wang Q, Mao WM, et al. Gefitinib versus vinorelbine plus cisplatin as adjuvant treatment for stage II-IIIA (N1-N2) EGFR-mutant NSCLC (ADJUVANT/ CTONG1104): a randomised, open-label, phase 3 study. Lancet Oncol 2018;19:139-48.

13. D'Angelo SP, Janjigian YY, Ahye N, et al. Distinct clinical course of EGFR-mutant resected lung cancers: results of testing of 1118 surgical specimens and effects of adjuvant gefitinib and erlotinib. J Thorac Oncol 2012;7:1815-22.

14. Janjigian YY, Park BJ, Zakowski MF, et al. Impact on disease-free survival of adjuvant erlotinib or gefitinib in patients with resected lung adenocarcinomas that harbor EGFR mutations. J Thorac Oncol 2011;6:569-75.

15. Pennell NA, Neal JW, Chaft JE, et al. SELECT: A Phase II Trial of Adjuvant Erlotinib in Patients With Resected Epidermal Growth Factor Receptor-Mutant Non-SmallCell Lung Cancer. J Clin Oncol 2019;37:97-104.

16. Liang W, Cai K, Chen C, et al. Society for Translational Medicine consensus on postoperative management of EGFR-mutant lung cancer (2019 edition). Transl Lung Cancer Res 2019;8:1163-73. 